in mass-casualty, ship accidents.

Methods: 18 members of the FGN Weapons Divers Group were included in a two-rescuer CPR mannequin training course with an AMBU CPR trainer connected to a volumeter. After video demonstration a modified LAR V or an AMBU MARK III bag had to be used for artificial mask-ventilation in a randomized, crossover design. During 10 cycles of CPR, tidal volumes were measured. CPR performance was analyzed using video tape recordings. The exact two-tailed Wilcoxon matched-pairs test was used for statistical analysis.

Results: All but one of the persons tested were able to ventilate the mannequin using the LAR V. Median tidal volumes were lower with LAR V vs. AMBU $(725 \mathrm{ml}$ vs. $800 \mathrm{ml} ; p=0.04$ ). In 84 vs. 58 (ns) ventilations, a tidal volume below $700 \mathrm{ml}$ was administered. In 45 vs. 15 cases, could be attributed to difficulties providing a leakproof seal to the face. The median total time required for 10 cycles of CPR was significantly longer with use of the LAR $V$ than the AMBU (90s vs. 68.5 seconds; $p=$ 0.004 ), mainly caused by the rescuer waiting for re-inflation of the LAR V reservoir bag until the respiratory cycle was started. Stomach insufflation was caused only by AMBU (18 ventilations, 4 rescuers).

Conclusion: This modification of the LAR V makes it suitable for CPR performed by military divers. Integration of a T-piece and a face mask into a military diver's survival kit could be helpful when CPR is required and the number of casualties exceeds the number of ventilatory devices.

Key Words: mass casualty ship accidents; underwater breathing apparatus; ventilatory device

\section{Did Cardio-Pulmonary Resuscitation Already Exist in Egypt 5,000 Years Ago? Andreas Ocklitz}

Anästhesiologie, St. Gertrauden Krankenhaus, Berlin, Germany

In light of the medically relevant features of the ancient Egyptian mouth-opening ceremony, the question of the effectiveness of medical practices in Egypt thousands of years ago is examined, whereby the religious and cultural framework also plays a significant role. In the Land on the Nile myth and reality clearly generated special conditions that favored the systematic treatment of questions of resuscitation. Numerous examples show that this had practical consequences in the area of everyday medicine. In addition, rebirth and resurrection were central elements of the cult of the dead which had exact medical equivalents. These equivalents may demonstrate the advanced state of resuscitation practices in Egypt at that time.

In this context, a reconstruction of an ancient Egyptian mouth-opening instrument is presented. In the cult of the dead, this instrument played a role which can be compared to the function of a modern laryngoscope. It appears possible that at the time of the pyramids, the Egyptians already had an understanding of the technology required to perform instrument-aided artifi- cial respiration. Whether or not they actually possessed a fundamental knowledge of the principles of cardiopulmonary resuscitation remains unclear. Nevertheless, the astonishingly functional characteristics of the reconstructed mouth-opening instrument suggest that it was developed for more than purely symbolic purposes.

Key Words: cult of the dead; reconstruction of the mouth-opening instrument; resuscitation in ancient Egypt

\section{Eisenmenger's Biomotor- \\ Predecessor of ACD-CPR?}

K.P. Koetter; ${ }^{1}$ W.H. Maleck ${ }^{2}$

1. Juliusspital, Wurzburg, Germany 2. Klinikum, Ludwigshafen, Germany

Introduction: Lerman mentioned in 1994 Eisenmenger's Biomotor as a precursor of Active CompressionDecompression CardioPulmonary Resuscitation (ACDCPR).

Methods: We checked the 1939 publication of Eisenmenger cited by Lerman, the secondary literature there of, and the Index Medicus for the years 1939-1949 to find further evidence.

Results: Eisenmenger published in 1903 a "Device for Artificial Respiration" consisting of an air-tight, thoraco-abdominal shield and a foot-operated bellows for generation of alternating pressure and vacuum on the abdomen. He proposed use of the device for patients in cardiopulmonary arrest caused by drowning or intoxication. The device was patented, and in 1904, it was available commercially.

In 1911, he published a successful resuscitation after one hour of "Vacuum and Pressure Massage of the Abdomen" in a case of suicide attempted by hanging. However, diagnosis of cardiac arrest was only clinical. The foot-operated bellows was replaced by a crank handle in 1928, and an electromotor (hence "Biomotor") in 1929.

Experiments on dogs in cardiac arrest were published in 1929 and 1932. With the methods available at this time, not only normal tidal volumes and blood pressure, but also gas exchange (i.e., $\mathrm{CO}_{2}$ exhalation) and transport of intravenous dye to all parts of the body were shown. In the 1930s, several contributions by others were published. In 1939, an eight-part series (the publication cited by Lerman) was published that described the use of the device in several hospitals. Further papers on the Biomotor by Eisenmenger appeared until 1942. We could not find a publication of Eisenmenger in 1943-1949.

Conclusion: The Biomotor combined active decompression as in ACD-CPR with the circumferential pressure used in vest $\mathrm{CPR}$ and pressure on the abdomen as in abdominal counterpulsation CPR. If a functional Biomotor could be found, an experimental re-evaluation is warranted.

Key Words: cardiopulmonary resuscitation; history of medicine 